Acta Crystallographica Section E

Structure Reports

Online

ISSN 1600-5368

\section{Andrei S. Batsanov, Alistair J. Reid and Neil Cameron}

Department of Chemistry, University of Durham, South Road, Durham DH1 3LE, England

Correspondence e-mail:

a.s.batsanov@durham.ac.uk

\section{Key indicators}

Single-crystal X-ray study

$T=100 \mathrm{~K}$

Mean $\sigma(\mathrm{C}-\mathrm{C})=0.001 \AA$

$R$ factor $=0.039$

$w R$ factor $=0.114$

Data-to-parameter ratio $=28.8$

For details of how these key indicators were automatically derived from the article, see http://journals.iucr.org/e.
(C) 2005 International Union of Crystallography Printed in Great Britain - all rights reserved

\title{
2-tert-Butoxy-1-phenyl-1-(2,2,6,6-tetramethyl- piperidin-1-yloxy)ethane
}

The title compound, $\mathrm{C}_{21} \mathrm{H}_{35} \mathrm{NO}_{2}$, contains a piperidine ring in a chair conformation, with a pyramidal $\mathrm{N}$ atom and a single (exocyclic) $\mathrm{N}-\mathrm{O}$ bond in an equatorial orientation.

\section{Comment}

Nitroxide-mediated polymerization (NMP) has emerged in recent years as a successful controlled or 'living' radical polymerization technique which can be used to prepare polymers of target molecular weight, narrow polydispersity and complex architecture (block and graft copolymers, star polymers, etc.) (Matyjaszewski, 2003). Successful NMP requires the use of a monomolecular initiator, identified as an alkoxyamine, which is derived from a nitroxide. This alkoxyamine should be prepared separately and then added in a known concentration to the monomer to be polymerized. Synthetic routes to alkoxyamines include the trapping of alkyl radicals by free nitroxides at moderate temperatures (Braslau et al., 1997; Miura et al., 1998) and a catalytic route involving Mn-salen complexes $\left[\mathrm{H}_{2}\right.$ salen is bis(salicylidene)ethylenediamine; Dao et al., 1998]. The title compound, (I), has been synthesized in the course of these studies (Cameron et al., 2000).

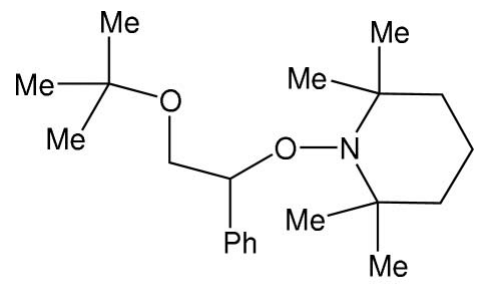

(I)

The molecular structure of (I) (Fig. 1) is similar in its main features to the other $\mathrm{N}$-oxy-2,2,6,6-tetramethylpiperidinyl derivatives reported by Ermert \& Vasella (1993), Jahn et al. (2001, 2002) and Leitich et al. (2002). The piperidine ring adopts a chair conformation with the $\mathrm{N}-\mathrm{O} 1$ bond in an equatorial orientation. The $\mathrm{N}-\mathrm{O} 1$ distance is typical for a single bond (Allen et al., 1987), while the $\mathrm{N}$ atom has pyramidal geometry, corresponding to $s p^{3}$ hybridization.

\section{Experimental}

A solution of 2,2,6,6-tetramethylpiperidinyloxy (TEMPO; $0.187 \mathrm{~g}$, $1.20 \mathrm{mmol})$ and di-tert-butylperoxalate $(0.148 \mathrm{~g}, 0.63 \mathrm{mmol})$ in styrene $(5 \mathrm{ml})$ was prepared. The mixture was degassed by three freeze/pump/thaw cycles and backflushed with argon after the final cycle. The resulting solution was sealed, then heated overnight at $313 \mathrm{~K}$. After cooling, the solution was dried in vacuo to constant
Received 21 January 2005 Accepted 14 February 2005 Online 19 February 2005 
mass. The crude solid product obtained was then purified by flash chromatography on silica gel, eluting with a mixture of 40-60 petroleum ether/ethyl acetate $(90: 10 v / v)$. After removal of the solvent, a white crystalline solid was obtained in $79 \%$ yield. The characterization data of the isolated product were in agreement with those given in the literature (Bon et al., 1999). ${ }^{1} \mathrm{H}$ NMR (300 MHz): $\delta$ $1.04\left(s, 9 \mathrm{H}, \mathrm{CH}_{3} \times 3\right), 0.58,1.02,1.19,1.37$ (br s, 3H, $\left.\mathrm{CH}_{3}\right), 0.9-1.7$ (br $\left.m, 6 \mathrm{H}, \mathrm{CH}_{2} \times 3\right), 3.41(m, 1 \mathrm{H}, \mathrm{H} 1), 3.89(m, 1 \mathrm{H}, \mathrm{H} 171), 4.74(m, 1 \mathrm{H}$, $\mathrm{H} 172), 7.18-7.35(m, 5 \mathrm{H}, \mathrm{Ph})$ p.p.m. (for the $\mathrm{H}$-atom numbering, see Fig. 1).

\section{Crystal data}

$$
\begin{aligned}
& \mathrm{C}_{21} \mathrm{H}_{35} \mathrm{NO}_{2} \\
& M_{r}=333.50 \\
& \text { Orthorhombic, } P b c a \\
& a=15.532(4) \AA \\
& b=11.119(3) \AA \\
& c=23.247(5) \AA \\
& V=4014.8(17) \AA^{3} \\
& Z=8 \\
& D_{x}=1.104 \mathrm{Mg} \mathrm{m}^{-3}
\end{aligned}
$$

Mo $K \alpha$ radiation

Cell parameters from 902 reflections

$\theta=12.1-24.3^{\circ}$

$\mu=0.07 \mathrm{~mm}^{-1}$

$T=100(2) \mathrm{K}$

Block, colourless

$1.00 \times 0.66 \times 0.14 \mathrm{~mm}$

\section{Data collection}

Bruker SMART 6000 CCD areadetector diffractometer

$\omega$ scans

Absorption correction: multi-scan (SADABS; Bruker, 2001)

$T_{\min }=0.579, T_{\max }=1.000$ 60196 measured reflections

\section{Refinement}

Refinement on $F^{2}$

$R\left[F^{2}>2 \sigma\left(F^{2}\right)\right]=0.039$

$w R\left(F^{2}\right)=0.114$

$S=1.06$

7229 reflections

251 parameters

$\mathrm{H}$ atoms treated by a mixture of independent and constrained refinement

Table 1

Selected geometric parameters $\left(\AA,^{\circ}\right)$.

\begin{tabular}{llll}
\hline $\mathrm{O} 1-\mathrm{C} 1$ & $1.4422(8)$ & $\mathrm{C} 2-\mathrm{C} 3$ & $1.5399(11)$ \\
$\mathrm{O} 1-\mathrm{N}$ & $1.4595(7)$ & $\mathrm{C} 3-\mathrm{C} 4$ & $1.5198(14)$ \\
$\mathrm{O} 2-\mathrm{C} 17$ & $1.4158(8)$ & $\mathrm{C} 4-\mathrm{C} 5$ & $1.5153(13)$ \\
$\mathrm{O} 2-\mathrm{C} 18$ & $1.4446(8)$ & $\mathrm{C} 5-\mathrm{C} 6$ & $1.5312(10)$ \\
$\mathrm{N}-\mathrm{C} 6$ & $1.4987(9)$ & $\mathrm{C} 6-\mathrm{C} 10$ & $1.5280(10)$ \\
$\mathrm{N}-\mathrm{C} 2$ & $1.5033(9)$ & $\mathrm{C} 6-\mathrm{C} 9$ & $1.5353(11)$ \\
$\mathrm{C} 1-\mathrm{C} 11$ & $1.5168(9)$ & $\mathrm{C} 18-\mathrm{C} 20$ & $1.5187(10)$ \\
$\mathrm{C} 1-\mathrm{C} 17$ & $1.5228(9)$ & $\mathrm{C} 18-\mathrm{C} 21$ & $1.5200(11)$ \\
$\mathrm{C} 2-\mathrm{C} 8$ & $1.5354(11)$ & $\mathrm{C} 18-\mathrm{C} 19$ & $1.5238(11)$ \\
$\mathrm{C} 2-\mathrm{C} 7$ & $1.5377(12)$ & & \\
$\mathrm{C} 1-\mathrm{O} 1-\mathrm{N}$ & $112.33(5)$ & $\mathrm{C} 5-\mathrm{C} 4-\mathrm{C} 3$ & $108.36(7)$ \\
$\mathrm{C} 17-\mathrm{O} 2-\mathrm{C} 18$ & $116.92(5)$ & $\mathrm{C} 4-\mathrm{C} 5-\mathrm{C} 6$ & $112.78(6)$ \\
$\mathrm{O} 1-\mathrm{N}-\mathrm{C} 6$ & $106.17(5)$ & $\mathrm{C} 10-\mathrm{C} 6-\mathrm{C} 5$ & $107.62(6)$ \\
$\mathrm{O} 1-\mathrm{N}-\mathrm{C} 2$ & $107.19(5)$ & $\mathrm{O} 2-\mathrm{C} 17-\mathrm{C} 1$ & $103.38(6)$ \\
$\mathrm{C} 6-\mathrm{N}-\mathrm{C} 2$ & $117.35(5)$ & $\mathrm{O} 2-\mathrm{C} 18-\mathrm{C} 20$ & $111.07(6)$ \\
$\mathrm{O} 1-\mathrm{C} 1-\mathrm{C} 11$ & $114.45(5)$ & $\mathrm{O} 2-\mathrm{C} 18-\mathrm{C} 21$ & $110.35(6)$ \\
$\mathrm{O} 1-\mathrm{C} 1-\mathrm{C} 17$ & $105.37(5)$ & $\mathrm{C} 20-\mathrm{C} 18-\mathrm{C} 21$ & $111.02(6)$ \\
$\mathrm{C} 11-\mathrm{C} 1-\mathrm{C} 17$ & $110.04(5)$ & $\mathrm{O} 2-\mathrm{C} 18-\mathrm{C} 19$ & $110.12(7)$ \\
$\mathrm{N}-\mathrm{C} 2-\mathrm{C} 3$ & $107.44(6)$ & $\mathrm{C} 20-\mathrm{C} 18-\mathrm{C} 19$ & $110.68(7)$ \\
$\mathrm{C} 4-\mathrm{C} 3-\mathrm{C} 2$ & $114.33(7)$ & $\mathrm{C} 21-\mathrm{C} 18-\mathrm{C} 19$ & \\
\hline
\end{tabular}
$R_{\text {int }}=0.020$

$\theta_{\text {max }}=32.5^{\circ}$

$h=-23 \rightarrow 23$

$k=-16 \rightarrow 16$

$l=-34 \rightarrow 35$

$$
\begin{gathered}
w=1 /\left[\sigma^{2}\left(F_{o}{ }^{2}\right)+(0.0633 P)^{2}\right. \\
\quad+0.7171 P] \\
\text { where } P=\left(F_{o}{ }^{2}+2 F_{c}{ }^{2}\right) / 3 \\
(\Delta / \sigma)_{\max }=0.001 \\
\Delta \rho_{\max }=0.52 \AA^{-3} \AA^{-3} \\
\Delta \rho_{\min }=-0.17 \mathrm{e}^{-3}
\end{gathered}
$$

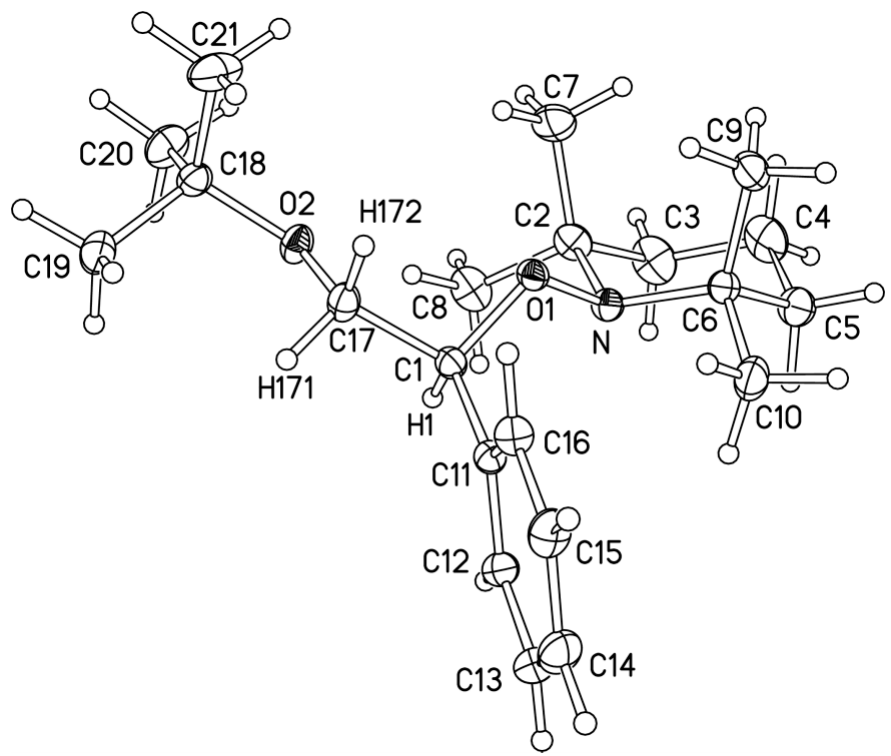

Figure 1

The molecular structure of (I), showing atomic displacement ellipsoids at the $50 \%$ probability level. 6298 reflections with $I>2 \sigma(I)$

Methyl groups were treated as threefold symmetrical bodies rotating around $\mathrm{C}-\mathrm{C}$ bonds, with a refined common $U_{\text {iso }}$ for the three $\mathrm{H}$ atoms. Other $\mathrm{H}$ atoms were treated as riding on the corresponding $\mathrm{C}$ atoms, with refined $U_{\text {iso }}$ values. $\mathrm{C}-\mathrm{H}$ distances are 0.95-1.00 ̊.

Data collection: SMART (Bruker, 2001); cell refinement: SMART; data reduction: SAINT (Bruker, 2001); program(s) used to solve structure: SHELXTL (Bruker, 2001); program(s) used to refine structure: SHELXTL; molecular graphics: SHELXTL; software used to prepare material for publication: SHELXTL.

\section{References}

Allen, F. H., Kennard, O., Watson, D. G., Brammer, L., Orpen, A. G. \& Taylor, R. (1987). J. Chem. Soc. Perkin Trans. 2, pp. S1-19.

Bon, S. A. F., Chambard, G. \& German, A. L. (1999). Macromolecules, 32, 8269-8276.

Braslau, R., Burrill, L. C., Siano, M., Naik, N., Howden, R. K. \& Mahal, L. K. (1997). Macromolecules, 30, 6445-6450.

Bruker (2001). SMART (Version 5.625), SAINT (Version 6.02A), SADABS (Version 2.03) and $S H E L X T L$ (Version 6.12). Bruker AXS Inc., Madison, Wisconsin, USA.

Cameron, N. R., Reid, A. J., Span, P., Bon, S. A. F., van Es, J. J. G. S. \& German, A. L. (2000). Macromol. Chem. Phys. 201, 2510-2518.

Dao, J., Benoit, D. \& Hawker, C. J. (1998). J. Polym. Sci. A, 36, 2161-2167.

Ermert, P. \& Vasella, A. (1993). Helv. Chim. Acta, 76, 2687-2699.

Jahn, U., Hartmann, P., Dix, I. \& Jones, P. G. (2001). Eur. J. Org. Chem. pp. 3333-3355.

Jahn, U., Hartmann, P., Dix, I. \& Jones, P. G. (2002). Eur. J. Org. Chem. pp. 718-735.

Leitich, J., Heize, I., Angermund, K. \& Rust, J. (2002). Eur. J. Org. Chem. pp. 1803-1825.

Matyjaszewski, K. (2003). Am. Chem. Soc. Symp. Ser. 854, $2-9$.

Miura, Y., Hirota, K., Moto, H. \& Yamada, B. (1998). Macromolecules, 31, 4659-4661. 


\title{
supporting information
}

Acta Cryst. (2005). E61, o716-o717 [https://doi.org/10.1107/S1600536805004976]

\section{2-tert-Butoxy-1-phenyl-1-(2,2,6,6-tetramethylpiperidin-1-yloxy)ethane}

\author{
Andrei S. Batsanov, Alistair J. Reid and Neil Cameron
}

2-tert-Butoxy-1-phenyl-1-(2,2,6,6-tetramethylpiperidin-1-yloxy)ethane

Crystal data

$\mathrm{C}_{21} \mathrm{H}_{35} \mathrm{NO}_{2}$

$M_{r}=333.50$

Orthorhombic, $\mathrm{Pbca}$

Hall symbol: -P 2ac $2 \mathrm{ab}$

$a=15.532(4) \AA$

$b=11.119(3) \AA$

$c=23.247(5) \AA$

$V=4014.8(17) \AA^{3}$

$Z=8$

\section{Data collection}

Bruker SMART 6000 CCD area-detector diffractometer

Radiation source: fine-focus sealed tube

Graphite monochromator

Detector resolution: 5.6 pixels $\mathrm{mm}^{-1}$

$\omega$ scans

Absorption correction: multi-scan

(SADABS; Bruker, 2001)

$T_{\min }=0.579, T_{\max }=1.000$

\section{Refinement}

Refinement on $F^{2}$

Least-squares matrix: full

$R\left[F^{2}>2 \sigma\left(F^{2}\right)\right]=0.039$

$w R\left(F^{2}\right)=0.114$

$S=1.06$

7229 reflections

251 parameters

0 restraints

Primary atom site location: structure-invariant direct methods
$F(000)=1472$

$D_{\mathrm{x}}=1.104 \mathrm{Mg} \mathrm{m}^{-3}$

Mo $K \alpha$ radiation, $\lambda=0.71073 \AA$

Cell parameters from 902 reflections

$\theta=12.1-24.3^{\circ}$

$\mu=0.07 \mathrm{~mm}^{-1}$

$T=100 \mathrm{~K}$

Block, colourless

$1.00 \times 0.66 \times 0.14 \mathrm{~mm}$

60196 measured reflections

7229 independent reflections

6298 reflections with $I>2 \sigma(I)$

$R_{\text {int }}=0.020$

$\theta_{\text {max }}=32.5^{\circ}, \theta_{\text {min }}=1.8^{\circ}$

$h=-23 \rightarrow 23$

$k=-16 \rightarrow 16$

$l=-34 \rightarrow 35$

Secondary atom site location: difference Fourier map

Hydrogen site location: difference Fourier map

$\mathrm{H}$ atoms treated by a mixture of independent and constrained refinement

$w=1 /\left[\sigma^{2}\left(F_{\mathrm{o}}^{2}\right)+(0.0633 P)^{2}+0.7171 P\right]$ where $P=\left(F_{\mathrm{o}}^{2}+2 F_{\mathrm{c}}^{2}\right) / 3$

$(\Delta / \sigma)_{\max }=0.001$

$\Delta \rho_{\max }=0.52$ e $\AA^{-3}$

$\Delta \rho_{\min }=-0.17$ e $\AA^{-3}$

\section{Special details}

Experimental. The data collection nominally covered full sphere of reciprocal space, by a combination of 4 sets of $\omega$ scans; each set at different $\varphi$ and/or $2 \theta$ angles and each scan $(20 \mathrm{sec}$ exposure $)$ covering $0.3^{\circ}$ in $\omega$. Crystal to detector distance $4.85 \mathrm{~cm}$.

Crystals are shattering when cut, therefore a crystal larger than the beam diameter was used and the intensities were corrected by $S A D A B S$ program (actual absorption is negligible). 
Geometry. All e.s.d.'s (except the e.s.d. in the dihedral angle between two 1.s. planes) are estimated using the full covariance matrix. The cell e.s.d.'s are taken into account individually in the estimation of e.s.d.'s in distances, angles and torsion angles; correlations between e.s.d.'s in cell parameters are only used when they are defined by crystal symmetry. An approximate (isotropic) treatment of cell e.s.d.'s is used for estimating e.s.d.'s involving 1.s. planes.

Refinement. Refinement of $F^{2}$ against ALL reflections. The weighted $R$-factor $w R$ and goodness of fit $S$ are based on $F^{2}$, conventional $R$-factors $R$ are based on $F$, with $F$ set to zero for negative $F^{2}$. The threshold expression of $F^{2}>\sigma\left(F^{2}\right)$ is used only for calculating $R$-factors(gt) etc. and is not relevant to the choice of reflections for refinement.

Fractional atomic coordinates and isotropic or equivalent isotropic displacement parameters $\left(\AA^{2}\right)$

\begin{tabular}{|c|c|c|c|c|}
\hline & $x$ & $y$ & $z$ & $U_{\text {iso }} * / U_{\text {eq }}$ \\
\hline $\mathrm{O} 1$ & $0.50726(3)$ & $0.34047(4)$ & $0.41290(2)$ & $0.01533(9)$ \\
\hline $\mathrm{O} 2$ & $0.33509(3)$ & $0.38797(5)$ & $0.37666(2)$ & $0.01928(10)$ \\
\hline $\mathrm{N}$ & $0.56521(4)$ & $0.25032(5)$ & $0.38875(2)$ & $0.01604(10)$ \\
\hline $\mathrm{C} 1$ & $0.43261(4)$ & $0.28730(6)$ & $0.43963(3)$ & $0.01552(11)$ \\
\hline H1 & 0.4152 & 0.2145 & 0.4172 & $0.016(2)^{*}$ \\
\hline $\mathrm{C} 2$ & $0.55469(5)$ & $0.25419(7)$ & $0.32449(3)$ & $0.02115(13)$ \\
\hline $\mathrm{C} 3$ & $0.62458(6)$ & $0.17267(8)$ & $0.29828(4)$ & $0.03198(17)$ \\
\hline H31 & 0.6109 & 0.0880 & 0.3078 & $0.040(3)^{*}$ \\
\hline H32 & 0.6228 & 0.1808 & 0.2559 & $0.044(3)^{*}$ \\
\hline $\mathrm{C} 4$ & $0.71538(6)$ & $0.20006(9)$ & $0.31880(4)$ & $0.03276(18)$ \\
\hline H41 & 0.7564 & 0.1415 & 0.3021 & $0.044(3)^{*}$ \\
\hline $\mathrm{H} 42$ & 0.7326 & 0.2818 & 0.3064 & $0.038(3)^{*}$ \\
\hline $\mathrm{C} 5$ & $0.71683(5)$ & $0.19182(7)$ & $0.38386(4)$ & $0.02631(15)$ \\
\hline H51 & 0.7755 & 0.2106 & 0.3977 & $0.032(3)^{*}$ \\
\hline H52 & 0.7032 & 0.1083 & 0.3955 & $0.035(3)^{*}$ \\
\hline C6 & $0.65274(4)$ & $0.27758(6)$ & $0.41254(3)$ & $0.01839(12)$ \\
\hline $\mathrm{C} 7$ & $0.55652(6)$ & $0.38093(8)$ & $0.29805(3)$ & $0.02855(16)$ \\
\hline H71 & $0.5145(5)$ & $0.4323(4)$ & $0.3179(3)$ & $0.0386(13)^{*}$ \\
\hline $\mathrm{H} 72$ & $0.5418(5)$ & $0.37606(12)$ & $0.2570(3)$ & $0.0386(13)^{*}$ \\
\hline H73 & $0.6144(4)$ & $0.4154(4)$ & $0.3024(3)$ & $0.0386(13)^{*}$ \\
\hline $\mathrm{C} 8$ & $0.46745(5)$ & $0.19737(8)$ & 0.30943 & $0.02742(15)$ \\
\hline H81 & $0.4625(2)$ & $0.1184(7)$ & $0.3284(3)$ & $0.0375(18)^{*}$ \\
\hline H82 & $0.4632(2)$ & $0.1870(6)$ & $0.2674(3)$ & $0.0375(18)^{*}$ \\
\hline H83 & $0.4207(3)$ & $0.2502(5)$ & 0.3228 & $0.0375(18)^{*}$ \\
\hline C9 & $0.68364(5)$ & $0.40811(7)$ & $0.40643(4)$ & $0.02434(14)$ \\
\hline H91 & $0.7018(5)$ & $0.4226(2)$ & $0.3668(3)$ & $0.0405(19)^{*}$ \\
\hline H92 & $0.7321(5)$ & $0.4220(2)$ & $0.4324(3)$ & $0.0405(19)^{*}$ \\
\hline H93 & $0.6367(4)$ & $0.4628(4)$ & $0.4162(3)$ & $0.0405(19)^{*}$ \\
\hline $\mathrm{C} 10$ & $0.65061(5)$ & $0.24755(8)$ & $0.47666(3)$ & $0.02721(15)$ \\
\hline H101 & $0.6137(5)$ & $0.3078(6)$ & $0.49731(15)$ & $0.0381(18)^{*}$ \\
\hline H102 & $0.7106(4)$ & $0.2503(7)$ & $0.49259(14)$ & $0.0381(18)^{*}$ \\
\hline H103 & $0.6261(5)$ & $0.1649(6)$ & $0.48219(6)$ & $0.0381(18)^{*}$ \\
\hline C11 & $0.44530(4)$ & $0.25205(6)$ & $0.50208(3)$ & $0.01565(11)$ \\
\hline $\mathrm{C} 12$ & $0.43806(4)$ & $0.13184(6)$ & $0.51850(3)$ & $0.01924(12)$ \\
\hline H12 & 0.4270 & 0.0723 & 0.4901 & $0.025(3)^{*}$ \\
\hline $\mathrm{C} 13$ & $0.44677(5)$ & $0.09773(7)$ & $0.57597(3)$ & $0.02422(14)$ \\
\hline H13 & 0.4423 & 0.0154 & 0.5865 & $0.033(3)^{*}$ \\
\hline
\end{tabular}




$\begin{array}{lllll}\text { C14 } & 0.46198(5) & 0.18453(8) & 0.61768(3) & 0.02565(15) \\ \text { H14 } & 0.4674 & 0.1618 & 0.6569 & 0.038(3)^{*} \\ \text { C15 } & 0.46920(5) & 0.30477(7) & 0.60196(3) & 0.02317(14) \\ \text { H15 } & 0.4796 & 0.3642 & 0.6305 & 0.036(3)^{*} \\ \text { C16 } & 0.46121(4) & 0.33830(6) & 0.54450(3) & 0.01906(12) \\ \text { H16 } & 0.4666 & 0.4205 & 0.5340 & 0.025(3)^{*} \\ \text { C17 } & 0.36194(4) & 0.38169(6) & 0.43478(3) & 0.01849(12) \\ \text { H171 } & 0.3127 & 0.3595 & 0.4596 & 0.024(2)^{*} \\ \text { H172 } & 0.3840 & 0.4609 & 0.4475 & 0.024(2)^{*} \\ \text { C18 } & 0.26850(4) & 0.47410(6) & 0.36304(3) & 0.01854(12) \\ \text { C19 } & 0.18898(5) & 0.45399(8) & 0.40020(4) & 0.02978(17) \\ \text { H191 } & 0.1726(3) & 0.3670(7) & 0.3991(3) & 0.0434(19)^{*} \\ \text { H192 } & 0.1403(4) & 0.5037(6) & 0.3852(2) & 0.0434(19)^{*} \\ \text { H193 } & 0.20186(18) & 0.4780(7) & 0.4408(3) & 0.0434(19)^{*} \\ \text { C20 } & 0.24793(6) & 0.44747(8) & 0.30046(3) & 0.02784(15) \\ \text { H201 } & 0.3015(4) & 0.4557(6) & 0.27679(17) & 0.0386(13)^{*} \\ \text { H202 } & 0.2035(4) & 0.5057(6) & 0.28632(14) & 0.0386(13)^{*} \\ \text { H203 } & 0.2252(5) & 0.3634(6) & 0.29697(6) & 0.0386(13)^{*} \\ \text { C21 } & 0.30130(6) & 0.60218(7) & 0.36949(4) & 0.03203(18) \\ \text { H211 } & 0.3145(5) & 0.6183(3) & 0.4105(3) & 0.045(2)^{*} \\ \text { H212 } & 0.2565(4) & 0.6593(4) & 0.3560(3) & 0.045(2)^{*} \\ \text { H213 } & 0.3542(5) & 0.6126(2) & 0.3461(3) & 0.045(2)^{*}\end{array}$

Atomic displacement parameters $\left(\AA^{2}\right)$

\begin{tabular}{lllllll}
\hline & $U^{11}$ & $U^{22}$ & $U^{33}$ & $U^{12}$ & $U^{13}$ & $U^{23}$ \\
\hline $\mathrm{O} 1$ & $0.01407(19)$ & $0.01375(19)$ & $0.0182(2)$ & $0.00091(15)$ & $0.00335(15)$ & $0.00001(15)$ \\
$\mathrm{O} 2$ & $0.0180(2)$ & $0.0243(2)$ & $0.0155(2)$ & $0.00446(17)$ & $-0.00207(16)$ & $0.00068(17)$ \\
$\mathrm{N}$ & $0.0160(2)$ & $0.0153(2)$ & $0.0169(2)$ & $0.00227(17)$ & $0.00319(17)$ & $-0.00101(17)$ \\
$\mathrm{C} 1$ & $0.0140(2)$ & $0.0174(3)$ & $0.0151(2)$ & $-0.00090(19)$ & $0.00074(18)$ & $0.0011(2)$ \\
$\mathrm{C} 2$ & $0.0229(3)$ & $0.0245(3)$ & $0.0160(3)$ & $-0.0010(2)$ & $0.0043(2)$ & $-0.0023(2)$ \\
$\mathrm{C} 3$ & $0.0312(4)$ & $0.0365(4)$ & $0.0283(4)$ & $0.0017(3)$ & $0.0104(3)$ & $-0.0114(3)$ \\
$\mathrm{C} 4$ & $0.0266(4)$ & $0.0364(4)$ & $0.0352(4)$ & $0.0043(3)$ & $0.0136(3)$ & $-0.0058(3)$ \\
$\mathrm{C} 5$ & $0.0201(3)$ & $0.0224(3)$ & $0.0365(4)$ & $0.0065(2)$ & $0.0073(3)$ & $0.0021(3)$ \\
$\mathrm{C} 6$ & $0.0149(2)$ & $0.0178(3)$ & $0.0225(3)$ & $0.0018(2)$ & $0.0017(2)$ & $0.0026(2)$ \\
$\mathrm{C} 7$ & $0.0322(4)$ & $0.0336(4)$ & $0.0199(3)$ & $-0.0027(3)$ & $0.0016(3)$ & $0.0078(3)$ \\
$\mathrm{C} 8$ & $0.0280(3)$ & $0.0342(4)$ & $0.0200(3)$ & $-0.0048(3)$ & $0.0009(3)$ & $-0.0077(3)$ \\
$\mathrm{C} 9$ & $0.0187(3)$ & $0.0198(3)$ & $0.0345(4)$ & $-0.0017(2)$ & $0.0003(3)$ & $-0.0003(3)$ \\
$\mathrm{C} 10$ & $0.0185(3)$ & $0.0396(4)$ & $0.0235(3)$ & $0.0032(3)$ & $-0.0020(2)$ & $0.0076(3)$ \\
$\mathrm{C} 11$ & $0.0140(2)$ & $0.0175(3)$ & $0.0155(2)$ & $0.0000(2)$ & $0.00058(19)$ & $0.00121(19)$ \\
$\mathrm{C} 12$ & $0.0195(3)$ & $0.0183(3)$ & $0.0199(3)$ & $-0.0009(2)$ & $-0.0012(2)$ & $0.0025(2)$ \\
$\mathrm{C} 13$ & $0.0243(3)$ & $0.0248(3)$ & $0.0235(3)$ & $0.0001(3)$ & $-0.0014(2)$ & $0.0078(2)$ \\
$\mathrm{C} 14$ & $0.0249(3)$ & $0.0343(4)$ & $0.0177(3)$ & $0.0030(3)$ & $-0.0017(2)$ & $0.0053(3)$ \\
$\mathrm{C} 15$ & $0.0228(3)$ & $0.0299(3)$ & $0.0169(3)$ & $0.0027(3)$ & $-0.0019(2)$ & $-0.0024(2)$ \\
$\mathrm{C} 16$ & $0.0191(3)$ & $0.0200(3)$ & $0.0181(3)$ & $0.0001(2)$ & $0.0001(2)$ & $-0.0011(2)$ \\
$\mathrm{C} 17$ & $0.0157(3)$ & $0.0247(3)$ & $0.0151(3)$ & $0.0034(2)$ & $-0.0002(2)$ & $0.0011(2)$ \\
$\mathrm{C} 18$ & $0.0189(3)$ & $0.0184(3)$ & $0.0184(3)$ & $0.0004(2)$ & $-0.0027(2)$ & $0.0026(2)$ \\
$\mathrm{C} 19$ & $0.0210(3)$ & $0.0351(4)$ & $0.0333(4)$ & $0.0090(3)$ & $0.0043(3)$ & $0.0111(3)$ \\
& & & & & &
\end{tabular}




$\begin{array}{lllllll}\mathrm{C} 20 & 0.0322(4) & 0.0299(4) & 0.0214(3) & 0.0024(3) & -0.0091(3) & 0.0007(3) \\ \mathrm{C} 21 & 0.0422(5) & 0.0208(3) & 0.0332(4) & -0.0059(3) & -0.0137(3) & 0.0053(3)\end{array}$

Geometric parameters $\left(\AA,{ }^{\circ}\right)$

\begin{tabular}{|c|c|c|c|}
\hline $\mathrm{O} 1-\mathrm{C} 1$ & $1.4422(8)$ & C9-H92 & 0.980 \\
\hline $\mathrm{O} 1-\mathrm{N}$ & $1.4595(7)$ & C9-H93 & 0.980 \\
\hline $\mathrm{O} 2-\mathrm{C} 17$ & $1.4158(8)$ & $\mathrm{C} 10-\mathrm{H} 101$ & 1.000 \\
\hline $\mathrm{O} 2-\mathrm{C} 18$ & $1.4446(8)$ & $\mathrm{C} 10-\mathrm{H} 102$ & 1.000 \\
\hline $\mathrm{N}-\mathrm{C} 6$ & $1.4987(9)$ & $\mathrm{C} 10-\mathrm{H} 103$ & 1.000 \\
\hline $\mathrm{N}-\mathrm{C} 2$ & $1.5033(9)$ & $\mathrm{C} 11-\mathrm{C} 12$ & $1.3945(10)$ \\
\hline $\mathrm{C} 1-\mathrm{C} 11$ & $1.5168(9)$ & $\mathrm{C} 11-\mathrm{C} 16$ & $1.3975(9)$ \\
\hline $\mathrm{C} 1-\mathrm{C} 17$ & $1.5228(9)$ & $\mathrm{C} 12-\mathrm{C} 13$ & $1.3955(10)$ \\
\hline $\mathrm{C} 1-\mathrm{H} 1$ & 1.000 & $\mathrm{C} 12-\mathrm{H} 12$ & 0.950 \\
\hline $\mathrm{C} 2-\mathrm{C} 8$ & $1.5354(11)$ & $\mathrm{C} 13-\mathrm{C} 14$ & $1.3883(12)$ \\
\hline $\mathrm{C} 2-\mathrm{C} 7$ & $1.5377(12)$ & $\mathrm{C} 13-\mathrm{H} 13$ & 0.950 \\
\hline $\mathrm{C} 2-\mathrm{C} 3$ & $1.5399(11)$ & $\mathrm{C} 14-\mathrm{C} 15$ & $1.3906(12)$ \\
\hline $\mathrm{C} 3-\mathrm{C} 4$ & $1.5198(14)$ & $\mathrm{C} 14-\mathrm{H} 14$ & 0.950 \\
\hline $\mathrm{C} 3-\mathrm{H} 31$ & 0.990 & $\mathrm{C} 15-\mathrm{C} 16$ & $1.3924(10)$ \\
\hline $\mathrm{C} 3-\mathrm{H} 32$ & 0.990 & C15-H15 & 0.950 \\
\hline $\mathrm{C} 4-\mathrm{C} 5$ & $1.5153(13)$ & $\mathrm{C} 16-\mathrm{H} 16$ & 0.950 \\
\hline $\mathrm{C} 4-\mathrm{H} 41$ & 0.990 & C17-H171 & 0.990 \\
\hline $\mathrm{C} 4-\mathrm{H} 42$ & 0.990 & C17-H172 & 0.990 \\
\hline $\mathrm{C} 5-\mathrm{C} 6$ & $1.5312(10)$ & $\mathrm{C} 18-\mathrm{C} 20$ & $1.5187(10)$ \\
\hline C5-H51 & 0.990 & $\mathrm{C} 18-\mathrm{C} 21$ & $1.5200(11)$ \\
\hline C5-H52 & 0.990 & $\mathrm{C} 18-\mathrm{C} 19$ & $1.5238(11)$ \\
\hline $\mathrm{C} 6-\mathrm{C} 10$ & $1.5280(10)$ & C19-H191 & 1.000 \\
\hline $\mathrm{C} 6-\mathrm{C} 9$ & $1.5353(11)$ & C19-H192 & 1.000 \\
\hline $\mathrm{C} 7-\mathrm{H} 71$ & 0.980 & C19-H193 & 1.000 \\
\hline $\mathrm{C} 7-\mathrm{H} 72$ & 0.980 & $\mathrm{C} 20-\mathrm{H} 201$ & 1.000 \\
\hline $\mathrm{C} 7-\mathrm{H} 73$ & 0.980 & $\mathrm{C} 20-\mathrm{H} 202$ & 1.000 \\
\hline $\mathrm{C} 8-\mathrm{H} 81$ & 0.990 & $\mathrm{C} 20-\mathrm{H} 203$ & 1.000 \\
\hline $\mathrm{C} 8-\mathrm{H} 82$ & 0.990 & $\mathrm{C} 21-\mathrm{H} 211$ & 0.990 \\
\hline $\mathrm{C} 8-\mathrm{H} 83$ & 0.990 & $\mathrm{C} 21-\mathrm{H} 212$ & 0.990 \\
\hline C9-H91 & 0.980 & $\mathrm{C} 21-\mathrm{H} 213$ & 0.990 \\
\hline $\mathrm{C} 1-\mathrm{O} 1-\mathrm{N}$ & $112.33(5)$ & $\mathrm{H} 91-\mathrm{C} 9-\mathrm{H} 93$ & 109.5 \\
\hline $\mathrm{C} 17-\mathrm{O} 2-\mathrm{C} 18$ & $116.92(5)$ & $\mathrm{H} 92-\mathrm{C} 9-\mathrm{H} 93$ & 109.5 \\
\hline $\mathrm{O} 1-\mathrm{N}-\mathrm{C} 6$ & $106.17(5)$ & $\mathrm{C} 6-\mathrm{C} 10-\mathrm{H} 101$ & 109.5 \\
\hline $\mathrm{O} 1-\mathrm{N}-\mathrm{C} 2$ & $107.19(5)$ & $\mathrm{C} 6-\mathrm{C} 10-\mathrm{H} 102$ & 109.5 \\
\hline $\mathrm{C} 6-\mathrm{N}-\mathrm{C} 2$ & $117.35(5)$ & $\mathrm{H} 101-\mathrm{C} 10-\mathrm{H} 102$ & 109.5 \\
\hline $\mathrm{O} 1-\mathrm{C} 1-\mathrm{C} 11$ & $114.45(5)$ & $\mathrm{C} 6-\mathrm{C} 10-\mathrm{H} 103$ & 109.5 \\
\hline $\mathrm{O} 1-\mathrm{C} 1-\mathrm{C} 17$ & $105.37(5)$ & $\mathrm{H} 101-\mathrm{C} 10-\mathrm{H} 103$ & 109.5 \\
\hline $\mathrm{C} 11-\mathrm{C} 1-\mathrm{C} 17$ & $110.04(5)$ & $\mathrm{H} 102-\mathrm{C} 10-\mathrm{H} 103$ & 109.5 \\
\hline $\mathrm{O} 1-\mathrm{C} 1-\mathrm{H} 1$ & 108.9 & $\mathrm{C} 12-\mathrm{C} 11-\mathrm{C} 16$ & $118.62(6)$ \\
\hline $\mathrm{C} 11-\mathrm{C} 1-\mathrm{H} 1$ & 108.9 & $\mathrm{C} 12-\mathrm{C} 11-\mathrm{C} 1$ & $119.94(6)$ \\
\hline $\mathrm{C} 17-\mathrm{C} 1-\mathrm{H} 1$ & 108.9 & $\mathrm{C} 16-\mathrm{C} 11-\mathrm{C} 1$ & $121.40(6)$ \\
\hline $\mathrm{N}-\mathrm{C} 2-\mathrm{C} 8$ & $108.10(5)$ & $\mathrm{C} 11-\mathrm{C} 12-\mathrm{C} 13$ & $120.97(7)$ \\
\hline
\end{tabular}




\begin{tabular}{|c|c|c|c|}
\hline $\mathrm{N}-\mathrm{C} 2-\mathrm{C} 7$ & $114.93(6)$ & $\mathrm{C} 11-\mathrm{C} 12-\mathrm{H} 12$ & 119.5 \\
\hline $\mathrm{C} 8-\mathrm{C} 2-\mathrm{C} 7$ & $107.59(7)$ & $\mathrm{C} 13-\mathrm{C} 12-\mathrm{H} 12$ & 119.5 \\
\hline $\mathrm{N}-\mathrm{C} 2-\mathrm{C} 3$ & $107.44(6)$ & $\mathrm{C} 14-\mathrm{C} 13-\mathrm{C} 12$ & $119.75(7)$ \\
\hline $\mathrm{C} 8-\mathrm{C} 2-\mathrm{C} 3$ & $106.83(6)$ & $\mathrm{C} 14-\mathrm{C} 13-\mathrm{H} 13$ & 120.1 \\
\hline $\mathrm{C} 7-\mathrm{C} 2-\mathrm{C} 3$ & $111.61(6)$ & $\mathrm{C} 12-\mathrm{C} 13-\mathrm{H} 13$ & 120.1 \\
\hline $\mathrm{C} 4-\mathrm{C} 3-\mathrm{C} 2$ & $114.33(7)$ & $\mathrm{C} 13-\mathrm{C} 14-\mathrm{C} 15$ & $119.90(7)$ \\
\hline $\mathrm{C} 4-\mathrm{C} 3-\mathrm{H} 31$ & 108.7 & $\mathrm{C} 13-\mathrm{C} 14-\mathrm{H} 14$ & 120.0 \\
\hline $\mathrm{C} 2-\mathrm{C} 3-\mathrm{H} 31$ & 108.7 & $\mathrm{C} 15-\mathrm{C} 14-\mathrm{H} 14$ & 120.1 \\
\hline $\mathrm{C} 4-\mathrm{C} 3-\mathrm{H} 32$ & 108.7 & $\mathrm{C} 14-\mathrm{C} 15-\mathrm{C} 16$ & $120.16(7)$ \\
\hline $\mathrm{C} 2-\mathrm{C} 3-\mathrm{H} 32$ & 108.7 & $\mathrm{C} 14-\mathrm{C} 15-\mathrm{H} 15$ & 119.9 \\
\hline $\mathrm{H} 31-\mathrm{C} 3-\mathrm{H} 32$ & 107.6 & $\mathrm{C} 16-\mathrm{C} 15-\mathrm{H} 15$ & 119.9 \\
\hline $\mathrm{C} 5-\mathrm{C} 4-\mathrm{C} 3$ & $108.36(7)$ & $\mathrm{C} 15-\mathrm{C} 16-\mathrm{C} 11$ & $120.59(7)$ \\
\hline $\mathrm{C} 5-\mathrm{C} 4-\mathrm{H} 41$ & 110.0 & $\mathrm{C} 15-\mathrm{C} 16-\mathrm{H} 16$ & 119.7 \\
\hline $\mathrm{C} 3-\mathrm{C} 4-\mathrm{H} 41$ & 110.0 & $\mathrm{C} 11-\mathrm{C} 16-\mathrm{H} 16$ & 119.7 \\
\hline $\mathrm{C} 5-\mathrm{C} 4-\mathrm{H} 42$ & 110.0 & $\mathrm{O} 2-\mathrm{C} 17-\mathrm{C} 1$ & $108.48(5)$ \\
\hline $\mathrm{C} 3-\mathrm{C} 4-\mathrm{H} 42$ & 110.0 & $\mathrm{O} 2-\mathrm{C} 17-\mathrm{H} 171$ & 110.0 \\
\hline $\mathrm{H} 41-\mathrm{C} 4-\mathrm{H} 42$ & 108.4 & $\mathrm{C} 1-\mathrm{C} 17-\mathrm{H} 171$ & 110.0 \\
\hline $\mathrm{C} 4-\mathrm{C} 5-\mathrm{C} 6$ & $112.78(6)$ & $\mathrm{O} 2-\mathrm{C} 17-\mathrm{H} 172$ & 110.0 \\
\hline $\mathrm{C} 4-\mathrm{C} 5-\mathrm{H} 51$ & 109.0 & $\mathrm{C} 1-\mathrm{C} 17-\mathrm{H} 172$ & 110.0 \\
\hline $\mathrm{C} 6-\mathrm{C} 5-\mathrm{H} 51$ & 109.0 & $\mathrm{H} 171-\mathrm{C} 17-\mathrm{H} 172$ & 108.4 \\
\hline $\mathrm{C} 4-\mathrm{C} 5-\mathrm{H} 52$ & 109.1 & $\mathrm{O} 2-\mathrm{C} 18-\mathrm{C} 20$ & $103.38(6)$ \\
\hline $\mathrm{C} 6-\mathrm{C} 5-\mathrm{H} 52$ & 109.0 & $\mathrm{O} 2-\mathrm{C} 18-\mathrm{C} 21$ & $111.07(6)$ \\
\hline $\mathrm{H} 51-\mathrm{C} 5-\mathrm{H} 52$ & 107.8 & $\mathrm{C} 20-\mathrm{C} 18-\mathrm{C} 21$ & $110.35(6)$ \\
\hline $\mathrm{N}-\mathrm{C} 6-\mathrm{C} 10$ & $107.23(5)$ & $\mathrm{O} 2-\mathrm{C} 18-\mathrm{C} 19$ & $111.02(6)$ \\
\hline $\mathrm{N}-\mathrm{C} 6-\mathrm{C} 5$ & $107.65(6)$ & $\mathrm{C} 20-\mathrm{C} 18-\mathrm{C} 19$ & $110.12(7)$ \\
\hline $\mathrm{C} 10-\mathrm{C} 6-\mathrm{C} 5$ & $107.62(6)$ & $\mathrm{C} 21-\mathrm{C} 18-\mathrm{C} 19$ & $110.68(7)$ \\
\hline $\mathrm{N}-\mathrm{C} 6-\mathrm{C} 9$ & $116.15(5)$ & $\mathrm{C} 18-\mathrm{C} 19-\mathrm{H} 191$ & 109.5 \\
\hline $\mathrm{C} 10-\mathrm{C} 6-\mathrm{C} 9$ & $107.67(6)$ & $\mathrm{C} 18-\mathrm{C} 19-\mathrm{H} 192$ & 109.5 \\
\hline $\mathrm{C} 5-\mathrm{C} 6-\mathrm{C} 9$ & $110.19(6)$ & $\mathrm{H} 191-\mathrm{C} 19-\mathrm{H} 192$ & 109.5 \\
\hline $\mathrm{C} 2-\mathrm{C} 7-\mathrm{H} 71$ & 109.5 & $\mathrm{C} 18-\mathrm{C} 19-\mathrm{H} 193$ & 109.5 \\
\hline $\mathrm{C} 2-\mathrm{C} 7-\mathrm{H} 72$ & 109.5 & $\mathrm{H} 191-\mathrm{C} 19-\mathrm{H} 193$ & 109.5 \\
\hline $\mathrm{H} 71-\mathrm{C} 7-\mathrm{H} 72$ & 109.5 & $\mathrm{H} 192-\mathrm{C} 19-\mathrm{H} 193$ & 109.5 \\
\hline $\mathrm{C} 2-\mathrm{C} 7-\mathrm{H} 73$ & 109.5 & $\mathrm{C} 18-\mathrm{C} 20-\mathrm{H} 201$ & 109.5 \\
\hline $\mathrm{H} 71-\mathrm{C} 7-\mathrm{H} 73$ & 109.5 & $\mathrm{C} 18-\mathrm{C} 20-\mathrm{H} 202$ & 109.5 \\
\hline $\mathrm{H} 72-\mathrm{C} 7-\mathrm{H} 73$ & 109.5 & $\mathrm{H} 201-\mathrm{C} 20-\mathrm{H} 202$ & 109.5 \\
\hline $\mathrm{C} 2-\mathrm{C} 8-\mathrm{H} 81$ & 109.5 & $\mathrm{C} 18-\mathrm{C} 20-\mathrm{H} 203$ & 109.5 \\
\hline $\mathrm{C} 2-\mathrm{C} 8-\mathrm{H} 82$ & 109.5 & $\mathrm{H} 201-\mathrm{C} 20-\mathrm{H} 203$ & 109.5 \\
\hline $\mathrm{H} 81-\mathrm{C} 8-\mathrm{H} 82$ & 109.5 & $\mathrm{H} 202-\mathrm{C} 20-\mathrm{H} 203$ & 109.5 \\
\hline $\mathrm{C} 2-\mathrm{C} 8-\mathrm{H} 83$ & 109.5 & $\mathrm{C} 18-\mathrm{C} 21-\mathrm{H} 211$ & 109.5 \\
\hline $\mathrm{H} 81-\mathrm{C} 8-\mathrm{H} 83$ & 109.5 & $\mathrm{C} 18-\mathrm{C} 21-\mathrm{H} 212$ & 109.5 \\
\hline $\mathrm{H} 82-\mathrm{C} 8-\mathrm{H} 83$ & 109.5 & $\mathrm{H} 211-\mathrm{C} 21-\mathrm{H} 212$ & 109.5 \\
\hline $\mathrm{C} 6-\mathrm{C} 9-\mathrm{H} 91$ & 109.5 & $\mathrm{C} 18-\mathrm{C} 21-\mathrm{H} 213$ & 109.5 \\
\hline $\mathrm{C} 6-\mathrm{C} 9-\mathrm{H} 92$ & 109.5 & $\mathrm{H} 211-\mathrm{C} 21-\mathrm{H} 213$ & 109.5 \\
\hline $\mathrm{H} 91-\mathrm{C} 9-\mathrm{H} 92$ & 109.5 & $\mathrm{H} 212-\mathrm{C} 21-\mathrm{H} 213$ & 109.5 \\
\hline $\mathrm{C} 6-\mathrm{C} 9-\mathrm{H} 93$ & 109.5 & & \\
\hline $\mathrm{C} 1-\mathrm{O} 1-\mathrm{N}-\mathrm{C} 6$ & $-128.03(5)$ & $\mathrm{O} 1-\mathrm{N}-\mathrm{C} 6-\mathrm{C} 5$ & $-174.94(5)$ \\
\hline $\mathrm{C} 1-\mathrm{O} 1-\mathrm{N}-\mathrm{C} 2$ & $105.78(6)$ & $\mathrm{C} 2-\mathrm{N}-\mathrm{C} 6-\mathrm{C} 5$ & $-55.17(7)$ \\
\hline
\end{tabular}




\begin{tabular}{ll}
$\mathrm{N}-\mathrm{O} 1-\mathrm{C} 1-\mathrm{C} 11$ & $86.82(6)$ \\
$\mathrm{N}-\mathrm{O} 1-\mathrm{C} 1-\mathrm{C} 17$ & $-152.16(5)$ \\
$\mathrm{O} 1-\mathrm{N}-\mathrm{C} 2-\mathrm{C} 8$ & $-72.99(7)$ \\
$\mathrm{C} 6-\mathrm{N}-\mathrm{C} 2-\mathrm{C} 8$ & $167.78(6)$ \\
$\mathrm{O} 1-\mathrm{N}-\mathrm{C} 2-\mathrm{C} 7$ & $47.18(7)$ \\
$\mathrm{C} 6-\mathrm{N}-\mathrm{C} 2-\mathrm{C} 7$ & $-72.05(8)$ \\
$\mathrm{O} 1-\mathrm{N}-\mathrm{C} 2-\mathrm{C} 3$ & $172.05(5)$ \\
$\mathrm{C} 6-\mathrm{N}-\mathrm{C} 2-\mathrm{C} 3$ & $52.82(8)$ \\
$\mathrm{N}-\mathrm{C} 2-\mathrm{C} 3-\mathrm{C} 4$ & $-52.39(9)$ \\
$\mathrm{C} 8-\mathrm{C} 2-\mathrm{C} 3-\mathrm{C} 4$ & $-168.19(7)$ \\
$\mathrm{C} 7-\mathrm{C} 2-\mathrm{C} 3-\mathrm{C} 4$ & $74.46(9)$ \\
$\mathrm{C} 2-\mathrm{C} 3-\mathrm{C} 4-\mathrm{C} 5$ & $56.39(10)$ \\
$\mathrm{C} 3-\mathrm{C} 4-\mathrm{C} 5-\mathrm{C} 6$ & $-57.99(9)$ \\
$\mathrm{O} 1-\mathrm{N}-\mathrm{C} 6-\mathrm{C} 10$ & $69.52(6)$ \\
$\mathrm{C} 2-\mathrm{N}-\mathrm{C} 6-\mathrm{C} 10$ & $-170.72(6)$ \\
\hline
\end{tabular}
$\mathrm{O} 1-\mathrm{N}-\mathrm{C} 6-\mathrm{C} 9$
$\mathrm{C} 2-\mathrm{N}-\mathrm{C} 6-\mathrm{C} 9$
$\mathrm{C} 4-\mathrm{C} 5-\mathrm{C} 6-\mathrm{N}$
$\mathrm{C} 4-\mathrm{C} 5-\mathrm{C} 6-\mathrm{C} 10$
$\mathrm{C} 4-\mathrm{C} 5-\mathrm{C} 6-\mathrm{C} 9$
$\mathrm{O} 1-\mathrm{C} 1-\mathrm{C} 11-\mathrm{C} 12$
$\mathrm{C} 17-\mathrm{C} 1-\mathrm{C} 11-\mathrm{C} 12$
$\mathrm{O} 1-\mathrm{C} 1-\mathrm{C} 11-\mathrm{C} 16$
$\mathrm{C} 18-\mathrm{O} 2-\mathrm{C} 17-\mathrm{C} 1$
$\mathrm{O} 1-\mathrm{C} 1-\mathrm{C} 17-\mathrm{O} 2$
$\mathrm{C} 11-\mathrm{C} 1-\mathrm{C} 17-\mathrm{O} 2$
$\mathrm{C} 17-\mathrm{O} 2-\mathrm{C} 18-\mathrm{C} 20$
$\mathrm{C} 17-\mathrm{O} 2-\mathrm{C} 18-\mathrm{C} 21$
$\mathrm{C} 17-\mathrm{O} 2-\mathrm{C} 18-\mathrm{C} 19$

$-50.91(7)$

$68.86(8)$

$56.34(8)$

$171.63(7)$

$-71.23(8)$

$-117.89(6)$

$123.70(7)$

$64.45(8)$

$-53.96(8)$

$-179.32(5)$

$72.26(6)$

$-163.88(5)$

$-173.50(6)$

$68.17(8)$

$-55.45(8)$ 